\title{
Impulsive Radiation from a Vertical Electric Dipole above an Imperfectly Conducting Surface
}

\author{
Mark C. H. Lam
}

\begin{abstract}
This paper presents an analytical benchmark for numerical methods employed to EM wave simulation with applications in antenna design and radio communication. Spacetime expressions for the impulsive wave fields generated by a vertical electric dipole situated above a planar conducting surface are derived. The EM properties of the imperfectly conductive material are represented by a surface impedance and translated to the wave motion via employing the local plane wave relation as the boundary condition. At the core of tackling the impedance boundary value problem is the derivation of the space-time reflected-wave Green's function with the aid of the extended Cagniard-de Hoop method. The solutions for the reflected wave fields open the door to analysis of their properties in the far, intermediate-, and near-field regions, in the dependence of the material's EM parameters. Numerical results illustrate time snaps of the reflected-wave Green's tensors and time traces of the measurable wave fields.
\end{abstract}

Index Terms-Impedance boundary condition, imperfectly conducting surface, impulsive wave reflection, vertical electric dipole.

\section{INTRODUCTION}

The concept of an impedance boundary, [1], [2], is useful for electromagnetic (EM) modelling tools, which target at wave simulation in geometrically complex-structured configurations characterized by imperfectly conductive materials. The approximating representation of conducting bodies via the surface impedance boundary condition (IBC), e.g. [3] [4], [5], [6], is convenient to avoid a costly fine discretization strategy in unstructured mesh grid modelling methods, such as the finite-element method [7]. Similar holds for the FDTD method [8], which in conjunction with the IBC, e.g. [9], [10], has found widespread applications in the modelling of thin conductive structures, e.g. [11], [12], [13], [14]. Applications are found in antenna simulation, micro-strip design, e.g. [15], [16], and on-chip interconnect modelling, e.g. [17], [18], [19]. In the basic scalar impedance boundary formulation, the material's EM properties are translated to the wave motion via employing the local plane wave relation between the electric field strength $\mathbf{E}$ and the magnetic field strength $\mathbf{H}$ as the boundary condition at the material's surface $\partial \mathcal{D}$. The timedomain IBC takes the general form

$$
\mathbf{n} \times \mathbf{E}(\mathbf{r}, t)=-\mathbf{n} \times\left[\mathbf{n} \times \int_{\tau=\infty}^{t} Z(t-\tau) \mathbf{H}(\mathbf{r}, \tau) \mathrm{d} \tau\right],
$$

for all $\mathbf{r} \in \partial \mathcal{D}$. The normal vector $\mathbf{n}$ is directed to the domain of computation, and $Z(t)$ is a spatially independent

Manuscript received June 9, 2011; revised XXXX xx, XXXX.

Mark C. H. Lam, 23 Jasmijnstraat, 4621 DA, Bergen op Zoom, Noord-Brabant, The Netherlands (e-mail: m_chlam@yahoo.com, website: www.markchlam.com). wave impedance of the investigated material. The EM wave fields interior to conductive materials such as metals are for some applications of minor interest, while the prediction of the reflection behavior of metallic structures is essential in e.g. antenna applications.

In this paper, the impulsive radiation from an electric dipole situated above an imperfectly conducting body is studied analytically, e.g. [20], in the time domain. To serve this purpose, the canonical problem of a planar boundary $\partial \mathcal{D}$ separating a homogeneous, isotropic, nonconducting medium filling half-space $\mathcal{D}_{1}$ from a homogeneous, isotropic, conducting medium filling half-space $\mathcal{D}_{2}$, here called the second medium, is investigated. In contrast to [21], the impedance boundary approximation reduces the two-media wave problem to a half-space problem, in which the EM wave fields in $\mathcal{D}_{1}$ are of interest. The vertical electric dipole (VED) directed normally away from $\partial \mathcal{D}$ is investigated, whereas the angularly nonsymmetric case of a horizontal electric dipole directed tangentially to $\partial \mathcal{D}$ requires a separate study.

The objective of this paper is to derive space-time solutions for the impulsive EM wave fields after reflection against a planar impedance boundary. To this end, the Cagniard-de Hoop $(\mathrm{CdH})$ method is applied to furnishing the analytical transformation from the transform domain back to the time domain. The pioneering study of the $\mathrm{CdH}$ method employed to nonconducting two-media problems can be found in [22], in which the importance of using the causality-preserving unilateral Laplace transform was stressed. In the case the second medium is imperfectly conducting, the reflection coefficient depends on the Laplace parameter, for which situation an extension of the $\mathrm{CdH}$ method is required, e.g. [23], [24]. In [24], the Schouten-Van der Pol theorem from the unilateral Laplace transform [25], [26], [27], [28], was employed to reshaping the Laplace-domain wave field solution to a form such that its time-domain counterpart can be found by simple inspection. Following that strategy in this paper, the space-time expression for the reflected-wave Green's function (RWGF) is analytically derived, from which the reflected-wave Green's tensor (RWGT) components are deduced.

This paper develops an analytical benchmark for numerical methods employed to EM wave simulation with applications in antenna design and radio communication. Furthermore, the presented space-time solutions for the reflected wave fields open the door to analysis of their properties in the far-, intermediate-, and near-field regions, in their dependence of the second medium's EM parameters. Illustrative numerical results present time snaps of the RWGT components to reveal the spatial properties of the reflected wave fields, which can only be obtained by analytical means. Time traces of the 
measurable EM wave fields are included to serve as benchmark results.

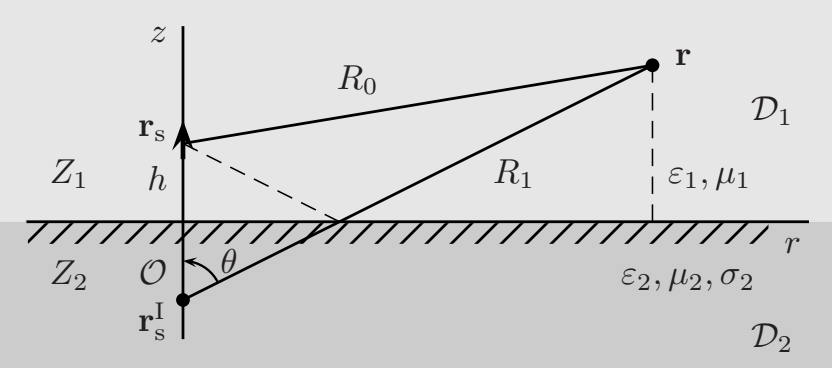

Fig. 1. Elementary configuration consisting of a planar interface $\partial \mathcal{D}$ separating two media. Half-space $\mathcal{D}_{1}$ is homogeneous, nonconducting and characterized by the wave impedance $Z_{1}$, whereas half-space $\mathcal{D}_{2}$ is homogeneous, imperfectly conducting and characterized by the wave impedance $Z_{2}$. With the surface impedance boundary condition, the electric properties in $\mathcal{D}_{2}$, called second medium, are represented at $\partial \mathcal{D}$. The symbol $\mathbf{r}_{\mathrm{s}}$ denotes the dipole source position, $\mathbf{r}_{\mathrm{s}}^{\mathrm{I}}$ the image source position, and $h$ the source height. Furthermore, $R_{0}$ is the distance between source and receiver, and $R_{1}$ is the distance between image source and receiver.

\section{Problem FORMUlation}

The electromagnetic wave motion is studied in the homogeneous half-space $\mathcal{D}_{1}=\{-\infty<x<\infty,-\infty<y<$ $\infty, 0<z<\infty\}$, which is characterized by the constant electric permittivity $\varepsilon_{1}$ and magnetic permeability $\mu_{1}$. The EM wave speed is consequently $c_{1}=\left(\varepsilon_{1} \mu_{1}\right)^{-1 / 2}$. Figure 1 shows the configuration including the nomenclature. The space-time EM wave fields in $\mathcal{D}_{1}$ satisfy the Maxwell's equations

$$
\begin{aligned}
-\operatorname{curl} \mathbf{H}+\varepsilon_{1} \partial_{t} \mathbf{E} & =-\mathbf{J}^{\mathrm{e}}, \\
\operatorname{curl} \mathbf{E}+\mu_{1} \partial_{t} \mathbf{H} & =-\mathbf{K}^{\mathrm{e}},
\end{aligned}
$$

in which

$$
\left\{\mathbf{J}^{\mathrm{e}}, \mathbf{K}^{\mathrm{e}}\right\}(\mathbf{r}, t)=\left\{J^{\mathrm{e}}, 0\right\}(t) \delta\left(\mathbf{r}-\mathbf{r}_{\mathrm{s}}\right) \mathbf{i}_{z},
$$

are the volume density of externally applied electric and magnetic current density, respectively. The symbol $J^{\mathrm{e}}$ stands for the source signature, $\delta(\mathbf{r})$ is the Kronecker delta function operative at $\mathbf{r}$, and $\mathbf{r}_{\mathrm{s}}=(0,0, h)$ denotes the position of the point source, with $h \geq 0$ the source height. The source starts to act at $t=0$ and prior to this time instant the configuration is at rest. Note that the dipole is directed perpendicularly away from the planar conducting surface, aligned with the normal vector $\mathbf{n}$ of $\partial \mathcal{D}$.

Let $Y_{1}=\left(\varepsilon_{1} / \mu_{1}\right)^{1 / 2}$ and $Z_{1}=\left(\mu_{1} / \varepsilon_{1}\right)^{1 / 2}$ denote the wave admittance and wave impedance of $\mathcal{D}_{1}$, respectively. Furthermore, let $Y_{\mathrm{n}}(t)$ and $Z_{\mathrm{n}}(t)$ stand for the specific wave admittance and the specific wave impedance of the second medium in $\mathcal{D}_{2}$, respectively. Specific means normalized with respect to its counterpart in $\mathcal{D}_{1}$. Then, the EM properties of the planar boundary are modelled via the linear, time-invariant, local admittance relation,

$$
\mathbf{H}_{\mathrm{T}}(x, y, 0, t)=-Y_{2}(t) \stackrel{(t)}{*}\left[\mathbf{E}_{\mathrm{T}}(x, y, 0, t) \times \mathbf{n}\right],
$$

in which $Y_{2}(t)=Y_{1} Y_{\mathrm{n}}(t)$ is the wave admittance of $\mathcal{D}_{2}$, or the local impedance relation

$$
\mathbf{E}_{\mathrm{T}}(x, y, 0, t)=-Z_{2}(t) \stackrel{(t)}{*}\left[\mathbf{n} \times \mathbf{H}_{\mathrm{T}}(x, y, 0, t)\right],
$$

in which $Z_{2}(t)=Z_{1} Z_{\mathrm{n}}(t)$ is the wave impedance of $\mathcal{D}_{2}$. In these expressions, the operation $\stackrel{(t)}{*}$ denotes temporal convolution and the subscript $\mathrm{T}$ stands for the tangential component. The EM wave field in $\mathcal{D}_{1}$ is defined as the sum of the incident wave field $\left\{\mathbf{E}^{\mathrm{inc}}, \mathbf{H}^{\mathrm{inc}}\right\}$, which is the wave field in the absence of the lossy material filling $\mathcal{D}_{2}$, and the reflected wave field $\left\{\mathbf{E}^{\text {ref }}, \mathbf{H}^{\text {ref }}\right\}$, which expresses the presence of the planar boundary,

$$
\{\mathbf{E}, \mathbf{H}\}(\mathbf{r}, t)=\left\{\mathbf{E}^{\text {inc }}, \mathbf{H}^{\text {inc }}\right\}(\mathbf{r}, t)+\left\{\mathbf{E}^{\text {ref }}, \mathbf{H}^{\text {ref }}\right\}(\mathbf{r}, t) .
$$

The focus in the remainder is on the derivation of the spacetime reflected EM wave fields in $\mathcal{D}_{1}$.

The time-invariance and causality of the EM wave fields are taken into account by the use of the unilateral Laplace transform

$$
\{\hat{\mathbf{E}}, \hat{\mathbf{H}}\}(\mathbf{r}, s)=\int_{t=0}^{\infty} \exp (-s t)\{\mathbf{E}, \mathbf{H}\}(\mathbf{r}, t) \mathrm{d} t,
$$

in which the Laplace transform parameter $s$ is taken positive and real. According to Lerch's theorem [29], a one-to-one mapping exists then between $\{\mathbf{E}, \mathbf{H}\}(\mathbf{r}, t)$ and their Laplace transformed counterparts $\{\hat{\mathbf{E}}, \hat{\mathbf{H}}\}(\mathbf{r}, s)$. The configuration is initially at rest, so the transform rule $\partial_{t} \rightarrow s$ holds. The complex slowness representation for $\{\hat{\mathbf{E}}, \hat{\mathbf{H}}\}(\mathbf{r}, s)$ are introduced as

$$
\begin{gathered}
\{\hat{\mathbf{E}}, \hat{\mathbf{H}}\}(\mathbf{r}, s)=\frac{s^{2}}{4 \pi^{2}} \int_{\alpha=-\infty}^{\infty} \mathrm{d} \alpha \\
\int_{\beta=-\infty}^{\infty}\{\tilde{\mathbf{E}}, \tilde{\mathbf{H}}\}(\alpha, \beta, z, s) \exp [-\mathrm{i} s(\alpha x+\beta y)] \mathrm{d} \beta,
\end{gathered}
$$

in which i stands for the imaginary unit, and $\alpha$ and $\beta$ denote the wave slowness in the $x$ and $y$ directions, respectively. The transform rules $\partial_{x} \rightarrow-\mathrm{i} s \alpha$ and $\partial_{y} \rightarrow-\mathrm{i} s \beta$ apply.

\section{THE REFLECTED ELECTROMAGNETIC WAVE FIELDS}

\section{A. The space-time structure}

Let $R_{1}(\mathbf{r})=\left[x^{2}+y^{2}+(z+h)^{2}\right]^{1 / 2}>0$ denote the distance between image source and receiver, and $T^{\mathrm{ref}}(\mathbf{r})=R_{1}(\mathbf{r}) / c_{1}$ the arrival time of the reflected wave. The reflected-wave Green's function is first written as $\mathcal{G}^{\mathrm{ref}}(\mathbf{r}, t)=G^{\mathrm{ref}}(\mathbf{r}, t) H[t-$ $T^{\mathrm{ref}}(\mathbf{r})$ ], in which $H(t)$ stands for the Heaviside unit step function. Then, the reflected wave field expressions corresponding to the incidence of a vertical electric dipole excitated spherical wave are given by (e.g. [30])

$$
\begin{aligned}
E_{x}^{\mathrm{ref}}(\mathbf{r}, t) & =-\varepsilon_{1}^{-1} J(t) \stackrel{(t)}{*} \partial_{x} \partial_{z} \mathcal{G}^{\mathrm{ref}}(\mathbf{r}, t), \\
E_{y}^{\mathrm{ref}}(\mathbf{r}, t)= & -\varepsilon_{1}^{-1} J(t) \stackrel{(t)}{*} \partial_{y} \partial_{z} \mathcal{G}^{\mathrm{ref}}(\mathbf{r}, t), \\
E_{z}^{\mathrm{ref}}(\mathbf{r}, t)= & -\varepsilon_{1}^{-1} J(t) \stackrel{(t)}{*} \partial_{z}^{2} \mathcal{G}^{\mathrm{ref}}(\mathbf{r}, t) \\
& +\mu_{1} \partial_{t}^{2} J(t) \stackrel{(t)}{*} \mathcal{G}^{\mathrm{ref}}(\mathbf{r}, t), \\
H_{x}^{\mathrm{ref}}(\mathbf{r}, t)= & +\partial_{t} J(t) \stackrel{(t)}{*} \partial_{y} \mathcal{G}^{\mathrm{ref}}(\mathbf{r}, t), \\
H_{y}^{\mathrm{ref}}(\mathbf{r}, t)= & -\partial_{t} J(t) \stackrel{(t)}{*} \partial_{x} \mathcal{G}^{\mathrm{ref}}(\mathbf{r}, t), \\
H_{z}^{\mathrm{ref}}(\mathbf{r}, t)= & 0 .
\end{aligned}
$$


Solving the canonical half-space problem is reduced to finding the space-time RWGF, to which end the inverse Laplace transformation of

$$
\begin{aligned}
& \hat{\mathcal{G}}^{\text {ref }}(\mathbf{r}, s)=\frac{1}{4 \pi^{2}} \int_{\alpha=-\infty}^{\infty} \mathrm{d} \alpha \\
& \int_{\beta=-\infty}^{\infty} \frac{\tilde{R}(\gamma, s)}{2 \gamma} \exp \left\{-s\left[\mathrm{i}(\alpha x+\beta y)+\gamma D_{z}\right]\right\} \mathrm{d} \beta
\end{aligned}
$$

is required. The reflection coefficient is here given by

$$
\tilde{R}(\gamma, s)=1-2 /\left[1+c_{1} \gamma \hat{Y}_{\mathrm{n}}(s)\right],
$$

in which $\gamma(\alpha, \beta)=\left(c_{1}^{-2}+\alpha^{2}+\beta^{2}\right)^{1 / 2}$ is the vertical slowness and

$$
\hat{Y}_{\mathrm{n}}(s)=\hat{X}_{\mathrm{Y}}^{1 / 2}(s)=F\left(1+\tau_{\text {rel }}^{-1} s^{-1}\right)^{1 / 2},
$$

is the plane wave specific admittance of the second medium in $\mathcal{D}_{2}$. Furthermore, $F=\left(\varepsilon_{2} / \varepsilon_{1}\right)^{1 / 2}\left(\mu_{1} / \mu_{2}\right)^{1 / 2}$ and $\tau_{\text {rel }}=$ $\varepsilon_{2} / \sigma_{2}$ stands for the second medium's relaxation time constant. Note that $\tilde{R}=1$, if either $F \rightarrow \infty$ or $\sigma_{2} \rightarrow \infty$, which cases lead to a perfectly reflecting boundary. The Laplacedomain specific impedance function is the inverse of Laplacedomain specific admittance function, i.e. $\hat{Z}_{\mathrm{n}}(s)=1 / \hat{Y}_{\mathrm{n}}(s)$. The space-time RWGF is presented next.

\section{B. Space-time reflected-wave Green's function}

The inverse Laplace transform of Eq. (16) can be derived with the aid of the extended $\mathrm{CdH}$ method, cf. [23], [24]. After transformation back to the time domain, it can be shown that the space-time RWGF can be expressed as

$$
\mathcal{G}^{\mathrm{ref}}(\mathbf{r}, t)=\mathcal{G}^{\mathrm{ref}, 1}(\mathbf{r}, t)+\mathcal{G}^{\mathrm{ref}, 2}(\mathbf{r}, t) \text {, for } \mathbf{r} \in \mathcal{D}_{1} .
$$

The first term on the r.h.s. is the constant-admittance RWGF, i.e. the special case of $\sigma_{2}=0 \mathrm{Sm}^{-1}$, and is given by

$$
\begin{aligned}
& \mathcal{G}^{\mathrm{ref}, 1}(\mathbf{r}, t)=\frac{1}{4 \pi R_{1}} H\left[t-T^{\mathrm{ref}}(\mathbf{r})\right] \\
& -\frac{1}{4 \pi R_{1}} \frac{2}{\left[\left(\Gamma_{1}^{2}+\Gamma_{2}^{2}\right) F^{2}+2 \Gamma_{1} F+1\right]^{1 / 2}} H\left[t-T^{\mathrm{ref}}(\mathbf{r})\right],
\end{aligned}
$$

in which

$$
\begin{aligned}
& \Gamma_{1}(\mathbf{r}, t)=\left(t / T^{\mathrm{ref}}\right) \cos (\theta), \\
& \Gamma_{2}(\mathbf{r}, t)=\left[\left(t / T^{\mathrm{ref}}\right)^{2}-1\right]^{1 / 2} \sin (\theta) .
\end{aligned}
$$

The second term on the r.h.s. of Eq. (19) takes into account the deviation from the case $\sigma_{2}=0$, and is given by

$$
\begin{gathered}
\mathcal{G}^{\mathrm{ref}, 2}(\mathbf{r}, t)=\frac{1}{4 \pi R_{1}} H\left[t-T^{\mathrm{ref}}(\mathbf{r})\right] \times \\
2 F \int_{\tau=T^{\mathrm{ref}}}^{t} \mathrm{~d} \tau \int_{v=0}^{\infty} \mathrm{d} v \int_{w=0}^{\infty} \mathrm{d} w K_{\mathrm{C}}(\mathbf{r}, \tau, v) \Psi(v, w) \times \\
\quad \exp \left(-w F^{2}\right)\left(\frac{w / \tau_{\mathrm{rel}}}{t-\tau}\right)^{1 / 2} J_{1}\left\{2 F\left[(t-\tau) w / \tau_{\mathrm{rel}}\right]^{1 / 2}\right\},
\end{gathered}
$$

in which $J_{1}$ stands for the Bessel function of the first kind and order one, and

$$
\Psi(v, w)=\frac{v}{\left(4 \pi w^{3}\right)^{1 / 2}} \exp \left(-v^{2} / 4 w\right) H(w),
$$

is the kernel function well-known from the theory of partial differential equations on diffusion processes, e.g. [31]. Furthermore, $K_{\mathrm{C}}(\mathbf{r}, \tau, v)$ is a kernel function that depends only on the configurational parameters, viz.,

$$
K_{\mathrm{C}}(\mathbf{r}, \tau, v)=\Lambda_{3} \exp \left(-\Lambda_{1} v\right) J_{0}\left(\Lambda_{2} v\right) H(v)
$$

in which $J_{0}$ stands for the Bessel function of the first kind and order zero, and

$$
\begin{aligned}
\Lambda_{1}(\mathbf{r}, \tau) & =\Gamma_{1} /\left(\Gamma_{1}^{2}+\Gamma_{2}^{2}\right), \\
\Lambda_{2}(\mathbf{r}, \tau) & =\Gamma_{2} /\left(\Gamma_{1}^{2}+\Gamma_{2}^{2}\right), \\
\Lambda_{3}(\mathbf{r}, \tau) & =1 /\left(\Gamma_{1}^{2}+\Gamma_{2}^{2}\right)^{1 / 2} .
\end{aligned}
$$

The expression in Eq. (23) represents solely the dispersive EM response of the imperfectly conductive material to an incident EM wave pulse. Note that $\mathcal{G}^{\text {ref, }, 2}\left(\mathbf{r}, t=T^{\text {ref }}\right)=0$ for all $\mathbf{r} \in \mathcal{D}_{1}$. This observation simplifies significantly the results in the next section, where the RWGT components $\partial_{n} \mathcal{G}^{\text {ref }}(\mathbf{r}, t)$ and $\partial_{m} \partial_{n} \mathcal{G}^{\text {ref }}(\mathbf{r}, t)$, are constructed from Eq. (19).

The mathematical derivation from Eq. (16) to Eqs. (19)(28) can be found in the Appendices A, B and C, where the extended $\mathrm{CdH}$ method is discussed. Finally, note that $\mathcal{G}^{\mathrm{ref}}(\mathbf{r}, t)=H\left(t-T^{\mathrm{ref}}\right) / 4 \pi R_{1}$ for $\tilde{R}=1$. This is the Green's function in homogeneous media, which was expected since the impedance boundary is in this special case a perfectly reflecting wall. For the nonconducting boundary case $\sigma_{2} \rightarrow 0$, the steady-state solution, i.e. $t \rightarrow \infty$, is $\mathcal{G}^{\text {ref }}(\mathbf{r}, t)=\mathcal{G}^{\text {ref, } 1}(\mathbf{r}, t) \rightarrow$ $H\left(t-T^{\mathrm{ref}}\right) / 4 \pi R_{1}$.

\section{SPATIAL DifFERENTIATION OF THE REFLECTED-WAVE GREEN'S FUNCTION}

In the numerical section, time snaps and time traces are illustrated in the $(x, z)$-plane. This choice is general since angular symmetry applies. Regarding the transverse components at this plane, only $E_{x}(\mathbf{r}, t)$ and $H_{y}(\mathbf{r}, t)$ are nonzero. To evaluate the space-time expressions for the reflected EM wave fields, the spatial differentiations in Eqs. (10)-(15) have to be evaluated. The jump discontinuity in the RWGF at the arrival time is taken care of by performing analytical differentiation as much as possible. After a lengthy derivation, including spatial differentiations of Eq. (19), the total-admittance reflected electric field strength $E_{x}^{\text {ref }}$ is found as

$$
E_{x}^{\mathrm{ref}}(\mathbf{r}, t)=E_{x}^{\mathrm{ref}, 1}(\mathbf{r}, t)+E_{x}^{\mathrm{ref}, 2}(\mathbf{r}, t),
$$

in which

$$
\begin{gathered}
E_{x}^{\mathrm{ref}, 1}(\mathbf{r}, t)=\varepsilon_{1}^{-1}\left(T^{\mathrm{ref}}\right)^{2} \partial_{t} J\left[t-T^{\mathrm{ref}}(\mathbf{r})\right] \mathcal{G}_{E_{x}}^{\mathrm{ref}, 1 ; \mathrm{FF}}(\mathbf{r}, t) \\
+\varepsilon_{1}^{-1} T^{\mathrm{ref}} J\left[t-T^{\mathrm{ref}}(\mathbf{r})\right] \mathcal{G}_{E_{x}}^{\mathrm{ref}, 1 ; \mathrm{IF}}(\mathbf{r}, t) \\
+\varepsilon_{1}^{-1} \int_{\tau=T^{\mathrm{ref}}}^{t} J(t-\tau) \mathcal{G}_{E_{x}}^{\mathrm{ref}, 1 ; \mathrm{NF}}(\mathbf{r}, \tau) \mathrm{d} \tau
\end{gathered}
$$

is the constant-admittance part of $E_{x}^{\mathrm{ref}}$, and

$$
E_{x}^{\mathrm{ref}, 2}(\mathbf{r}, t)=\varepsilon_{1}^{-1} \int_{\tau=T^{\mathrm{ref}}}^{t} J(t-\tau) \mathcal{G}_{E_{x}}^{\mathrm{ref}, 2 ; \mathrm{NF}}(\mathbf{r}, \tau) \mathrm{d} \tau,
$$


represents the dispersive part of $E_{x}^{\text {ref. }}$. The RWGT components in Eqs. (30)-(31) are given by

$$
\begin{gathered}
\mathcal{G}_{E_{x}}^{\mathrm{ref}, 1 ; \mathrm{FF}}(\mathbf{r}, t)=-\frac{x D_{z} G^{\mathrm{ref}, 1}\left[\mathbf{r}, T^{\mathrm{ref}}(\mathbf{r})\right]}{R_{1}^{4}} H\left[t-T^{\mathrm{ref}}(\mathbf{r})\right], \\
\mathcal{G}_{E_{x}}^{\mathrm{ref}, 1 ; \mathrm{IF}}(\mathbf{r}, t)=H\left[t-T^{\mathrm{ref}}(\mathbf{r})\right]\left\{\frac{x \partial_{z} G^{\mathrm{ref}, 1}\left[\mathbf{r}, T^{\mathrm{ref}}(\mathbf{r})\right]}{R_{1}^{2}}\right. \\
\left.\quad-\frac{x D_{z} G^{\mathrm{ref}, 1}\left[\mathbf{r}, T^{\mathrm{ref}}(\mathbf{r})\right]}{R_{1}^{4}}+\frac{D_{z} \partial_{x} G^{\mathrm{ref}, 1}\left[\mathbf{r}, T^{\mathrm{ref}}(\mathbf{r})\right]}{R_{1}^{2}}\right\},(33) \\
\mathcal{G}_{E_{x}}^{\mathrm{ref}, 1 ; \mathrm{NF}}(\mathbf{r}, t)=-\partial_{x} \partial_{z} G^{\mathrm{ref}, 1}(\mathbf{r}, t) H\left[t-T^{\mathrm{ref}}(\mathbf{r})\right], \\
\mathcal{G}_{E_{x}}^{\mathrm{ref}, 2 ; \mathrm{NF}}(\mathbf{r}, t)=-\partial_{x} \partial_{z} G^{\mathrm{ref}, 2}(\mathbf{r}, t) H\left[t-T^{\mathrm{ref}}(\mathbf{r})\right],
\end{gathered}
$$

in which the first two terms represent the far- and intermediatezone contributions, respectively, whereas the last two terms represent the near-zone contributions to $E_{x}^{\text {ref }}$. Similarly, the total-admittance reflected magnetic field strength $H_{y}^{\text {ref }}$ is found as

$$
H_{y}^{\mathrm{ref}}(\mathbf{r}, t)=H_{y}^{\mathrm{ref}, 1}(\mathbf{r}, t)+H_{y}^{\mathrm{ref}, 2}(\mathbf{r}, t),
$$

in which

$$
\begin{aligned}
& H_{y}^{\mathrm{ref}, 1}(\mathbf{r}, t)=T^{\mathrm{ref}} \partial_{t} J\left[t-T^{\mathrm{ref}}(\mathbf{r})\right] \mathcal{G}_{H_{y}}^{\mathrm{ref}, 1 ; \mathrm{FF}}(\mathbf{r}, t) \\
& \quad+\int_{\tau=T^{\mathrm{ref}}}^{t} \partial_{t} J(t-\tau) \mathcal{G}_{H_{y}}^{\mathrm{ref}, 1 ; \mathrm{IF}}(\mathbf{r}, \tau) \mathrm{d} \tau,
\end{aligned}
$$

is the constant-admittance part of $H_{y}^{\text {ref }}$, and

$$
H_{y}^{\mathrm{ref}, 2}(\mathbf{r}, t)=\int_{\tau=T^{\mathrm{ref}}}^{t} \partial_{t} J(t-\tau) \mathcal{G}_{H_{y}}^{\mathrm{ref}, 2 ; \mathrm{IF}}(\mathbf{r}, \tau) \mathrm{d} \tau,
$$

represents the dispersive part of $H_{y}^{\text {ref }}$. The RWGT components in Eqs. (37)-(38) are given by

$$
\begin{aligned}
\mathcal{G}_{H_{y}}^{\mathrm{ref}, 1 ; \mathrm{FF}}(\mathbf{r}, t) & =\left(x / R_{1}^{2}\right) G^{\mathrm{ref}, 1}\left[\mathbf{r}, T^{\mathrm{ref}}(\mathbf{r})\right] H\left[t-T^{\mathrm{ref}}(\mathbf{r})\right], \\
\mathcal{G}_{H_{y}}^{\mathrm{ref}, 1 ; \mathrm{IF}}(\mathbf{r}, t) & =-\partial_{x} G^{\mathrm{ref}, 1}(\mathbf{r}, t) H\left[t-T^{\mathrm{ref}}(\mathbf{r})\right], \\
\mathcal{G}_{H_{y}}^{\mathrm{ref}, 2 ; \mathrm{IF}}(\mathbf{r}, t) & =-\partial_{x} G^{\mathrm{ref}, 2}(\mathbf{r}, t) H\left[t-T^{\mathrm{ref}}(\mathbf{r})\right],
\end{aligned}
$$

in which the first term represents the far-zone contribution, whereas the last two terms represent the intermediate-zone contributions to $H_{y}^{\text {ref }}$.

The wave field decompositions for $E_{x}^{\text {ref }}$ and $H_{y}^{\text {ref }}$ reveal that the conductivity $\sigma_{2}$ is only present in Eqs. (35) and (41), which show that the dispersive EM response is only present in the near zone for $E_{x}^{\text {ref }}$, and in the intermediate zone for $H_{y}^{\text {ref }}$. This is the result of the identity $G^{\mathrm{ref}, 2}\left[\mathbf{r}, T^{\mathrm{ref}}(\mathbf{r})\right]=0$ for all $\mathbf{r} \in \mathcal{D}_{1}$. Note that $G^{\mathrm{ref}, 1}\left[\mathbf{r}, T^{\mathrm{ref}}(\mathbf{r})\right]$ is unequal to zero. The following expression is easily obtained from Eq. (20),

$$
G^{\mathrm{ref}, 1}\left[\mathbf{r}, T^{\mathrm{ref}}(\mathbf{r})\right]=\frac{1}{4 \pi R_{1}}\left[1-\frac{2}{F \cos (\theta)+1}\right],
$$

from which the analytical expressions

$$
\begin{aligned}
& \partial_{x} G^{\mathrm{ref}, 1}\left[\mathbf{r}, T^{\mathrm{ref}}(\mathbf{r})\right]=\frac{x}{4 \pi R_{1}^{3}}\left\{\frac{2}{[F \cos (\theta)+1]^{2}}-1\right\}, \\
& \partial_{y} G^{\mathrm{ref}, 1}\left[\mathbf{r}, T^{\mathrm{ref}}(\mathbf{r})\right]=\frac{y}{4 \pi R_{1}^{3}}\left\{\frac{2}{[F \cos (\theta)+1]^{2}}-1\right\}, \\
& \partial_{z} G^{\mathrm{ref}, 1}\left[\mathbf{r}, T^{\mathrm{ref}}(\mathbf{r})\right]= \\
& \frac{F+\cos (\theta)}{2 \pi R_{1}^{2}[F \cos (\theta)+1]^{2}}-\frac{\cos (\theta)}{4 \pi R_{1}^{2}},
\end{aligned}
$$

are derived. With these analytical expressions available, only Eqs. (34)-(35) and Eqs. (40)-(41) require numerical differentiation, which is performed with the first term of the Taylor expansion of the RWGF. For the perfectly reflecting boundary case $F \rightarrow \infty$, the results are $G^{\mathrm{ref}, 1}\left[\mathbf{r}, T^{\mathrm{ref}}(\mathbf{r})\right]=1 / 4 \pi R_{1}$, $\partial_{x} G^{\mathrm{ref}, 1}\left[\mathbf{r}, T^{\mathrm{ref}}(\mathbf{r})\right]=-x / 4 \pi R_{1}^{3}, \partial_{y} G^{\mathrm{ref}, 1}\left[\mathbf{r}, T^{\mathrm{ref}}(\mathbf{r})\right]=$ $-y / 4 \pi R_{1}^{3}$ and $\partial_{z} G^{\mathrm{ref}, 1}\left[\mathbf{r}, T^{\mathrm{ref}}(\mathbf{r})\right]=-D_{z} / 4 \pi R_{1}^{3}$, which accordingly are similar to the Green's function and Green's tensors in homogeneous media. Another check of correctness is presented in Appendix D, where the well-known solutions for the EM wave fields in homogeneous media are derived directly from Eqs. (29)-(41).
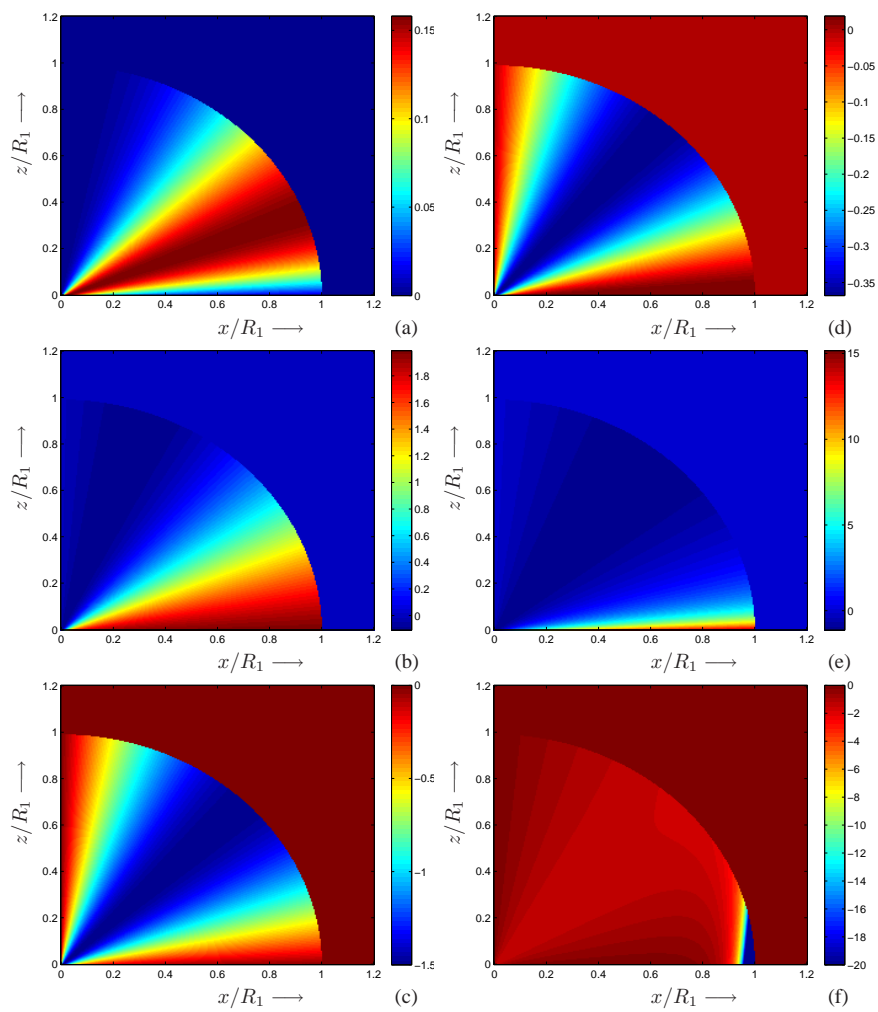

Fig. 2. Time snaps of the normalized RWGT components corresponding to $E_{x}^{\text {ref, } 1}(\mathbf{r}, t)$. (a) Far-zone RWGT component, $\left(4 \pi R_{1}^{3}\right) \times \mathcal{G}_{E_{x}}^{\text {ref }, 1 ; \mathrm{FF}}(\mathbf{r}, t)$, (b) intermediate-zone RWGT component, $\left(4 \pi R_{1}^{3}\right) \times \mathcal{G}_{E_{x}}^{\text {ref, } 1 ; \mathrm{IF}}(\mathbf{r}, t)$, and (c) nearzone RWGT component, $\left(4 \pi R_{1}^{3}\right) \times \mathcal{G}_{E_{x}}^{\text {ref, } 1 ; \mathrm{NF}}(\mathbf{r}, t)$, for copper as the second medium. (d)-(f) Same as (a)-(c), but now for sea water as the second medium.

\section{NumERicAl RESUlTS}

The impedance boundary concept is an accurate approximation if either $\sigma_{2} \gg 0$, or $\varepsilon_{2} \gg \varepsilon_{1}$. Both cases are studied simultaneously below, i.e., a metal and sea water are investigated, respectively. The magnetic permeability in both $\mathcal{D}_{1}$ and $\mathcal{D}_{2}$ is chosen $\mu_{1}=\mu_{2}=\mu_{0}$, i.e. the permeability of vacuum. The wave speed in domain $\mathcal{D}_{1}$ is in all examples taken $c_{1}=c_{0}$, which corresponds to $\varepsilon_{1}=\varepsilon_{0}$, where $\varepsilon_{0}=\left(c_{0}^{2} \mu_{0}\right)^{-1}$ is the electric permittivity of vacuum.

A source signature $J$ is specified for the computation of the wave field constituents. The source signature $J$ is taken

$$
J(t)= \begin{cases}0, & t<0 \\ \frac{\mathrm{d}}{\mathrm{d} t} W^{(m)}(t), & t \geq 0\end{cases}
$$

in which

$$
W^{(m)}(t)=W_{0}\left(t / \tau_{\mathrm{s}}\right)^{m} \exp \left[-m\left(t / \tau_{\mathrm{s}}-1\right)\right] H(t),
$$


is a $C^{m-1}$ power exponential function. The symbol $\tau_{\mathrm{s}}$ denotes the source signature's characterization time. The amplitude $W_{0}$ is chosen such that the maximum value of $J$ is unity. In the calculation of time traces, $m$ is taken four.
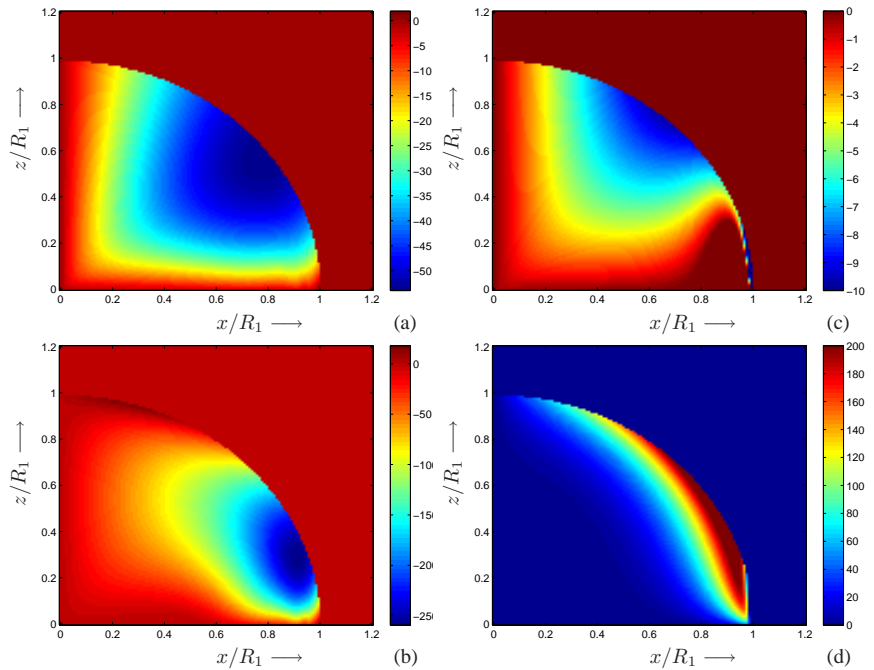

Fig. 3. Time snaps of the normalized RWGT components corresponding to $E_{x}^{\text {ref, }, 2}(\mathbf{r}, t)$. Near-zone RWGT component $\left(4 \pi R_{1}^{3}\right) \times \mathcal{G}_{E_{r}, 2 ; \mathrm{NF}}^{\text {ref }}(\mathbf{r}, t)$ at (a) $t=\tau_{\text {rel }}$ and (b) $t=8 \tau_{\text {rel }}$, for copper as the second medium. (c)-(d) Same as (a)-(b), but now for sea water as the second medium.

\section{A. Time snaps of the reflected-wave Green's tensors}

The RWGT components exhibit the spatial distribution of the reflected wave field constituents in the far-, intermediate, and near-field regions, and they are now investigated in the $(x, z)$-plane for $t$ fixed. The source height is taken $h=$ $R_{1} / 100$.

Figure 2(a)-(c) present time snaps of the normalized RWGT components corresponding to $E_{x}^{\mathrm{ref}, 1}(\mathbf{r}, t)$, i.e., (a) the far-zone RWGT $\left(4 \pi R_{1}^{3}\right) \times \mathcal{G}_{E_{x}}^{\text {ref }, 1 ; \mathrm{FF}}(\mathbf{r}, t)$, (b) the intermediate-zone RWGT $\left(4 \pi R_{1}^{3}\right) \times \mathcal{G}_{E_{x}}^{\text {ref, } 1 ; \mathrm{IF}}(\mathbf{r}, t)$, and (c) the near-zone RWGT $\left(4 \pi R_{1}^{3}\right) \times \mathcal{G}_{E_{x}}^{\text {ref }, 1 ; \mathrm{NF}}(\mathbf{r}, t)$, for copper as the second medium. Figure 2(d)-(f) present similar results as in Fig. 2(a)-(c), but now for sea water as the second medium. The dispersive part of the normalized RWGT components corresponding to $E_{x}^{\text {ref,2}}(\mathbf{r}, t)$ is shown in Fig. 3, i.e., the near-zone RWGT $\left(4 \pi R_{1}^{3}\right) \times \mathcal{G}_{E_{x}}^{\text {ref, } 2 ; \mathrm{NF}}(\mathbf{r}, t)$, at (a) $t=\tau_{\text {rel }}$, and (b) $t=8 \tau_{\text {rel }}$, respectively, for copper as the second medium. Figure 3(c)(d) present similar results as in Fig. 3(a)-(b), but now for sea water as the second medium.

Figure 4(a)-(b) present time snaps of the normalized RWGT components corresponding to $H_{y}^{\text {ref, }, 1}(\mathbf{r}, t)$, i.e., (a) the far-zone RWGT $\left(4 \pi R_{1}^{2}\right) \times \mathcal{G}_{H_{y}}^{\text {ref }, 1 ; \mathrm{FF}}(\mathbf{r}, t)$, and (b) the intermediatezone RWGT $\left(4 \pi R_{1}^{2}\right) \times \mathcal{G}_{H_{y}}^{\text {ref, } 1 \text { IF }}(\mathbf{r}, t)$, for copper as the second medium. Figure 4(c)-(d) present similar results as in Fig. 4(a)(b), but now for sea water as the second medium. The dispersive part of the normalized RWGT components corresponding to $H_{y}^{\text {ref,2 }}(\mathbf{r}, t)$ is shown in Fig. 5, i.e., the intermediate-zone RWGT $\left(4 \pi R_{1}^{2}\right) \times \mathcal{G}_{H_{y}}^{\text {ref, } 2 ; \mathrm{IF}}(\mathbf{r}, t)$, at (a) $t=\tau_{\text {rel }}$, and (b) $t=8 \tau_{\text {rel }}$, respectively, for copper as the second medium. Figure 5(c)-(d) present similar results as in Fig. 5(a)-(b), but now for sea water as the second medium.

\section{B. Time traces of the reflected electromagnetic wave fields}

Time traces for the reflected EM wave fields at the position $(x, y, z)=(2 h, 0, h)$, with $h=2 c_{1} \tau_{\text {rel }}$, are presented now for three different values of $\tau_{\mathrm{s}}$. It is convenient to scale both $E_{x}^{\mathrm{ref}}(\mathbf{r}, t)$ and $H_{y}^{\mathrm{ref}}(\mathbf{r}, t)$ to the same order of magnitude as the source signature $J$ for a direct check of correctness. Here, $E_{x}^{\mathrm{ref}}$ is displayed via

$$
\mathcal{E}_{x}^{\mathrm{ref}}(\mathbf{r}, t)=\left(4 \pi R_{1}^{3}\right) \times \varepsilon_{1} E_{x}^{\mathrm{ref}}(\mathbf{r}, t) / T^{\mathrm{ref}}(\mathbf{r}),
$$

and $H_{y}^{\text {ref }}$ is displayed via

$$
\mathcal{H}_{y}^{\mathrm{ref}}(\mathbf{r}, t)=\left(4 \pi R_{1}^{2}\right) \times H_{y}^{\mathrm{ref}}(\mathbf{r}, t) .
$$

These scalings yield values close to the amplitude of $J$, for all $\mathbf{r} \in \mathcal{D}_{1}$. Note that the scaling factors are independent of $t$. Time traces of $\mathcal{E}_{x}^{\text {ref }}(\mathbf{r}, t)$ are shown first. Figure 6(a)-(c) present modelling results corresponding to (a) $\tau_{\mathrm{s}}=0.5 \tau_{\text {rel }}$, (b) $\tau_{\mathrm{s}}=\tau_{\text {rel }}$, and (c) $\tau_{\mathrm{s}}=2 \tau_{\text {rel }}$, for copper as the second medium. Figure 6(d)-(f) present similar modelling results as in Fig. 6(a)-(c), but now for sea water as the second medium. Figure 7 presents the corresponding magnitude spectra, which are normalized with respect to $\max _{f}\left|\hat{\mathcal{E}}_{x}^{\text {ref }}(f)\right|$. Figures 8 and 9 present modelling results similar to Figs. 6 and 7, but now for $\mathcal{H}_{y}^{\mathrm{ref}}(\mathbf{r}, t)$.

In the late time, $\mathcal{G}^{\text {ref, } 1}(\mathbf{r}, t)$ goes to $H\left(t-T^{\text {ref }}\right) / 4 \pi R_{1}$ (since $\Gamma_{1}(\mathbf{r}, t)$ and $\Gamma_{2}(\mathbf{r}, t)$ in Eqs. (21)-(22) go to infinity as $t \rightarrow \infty)$, which allows analytical computation of $E^{\text {ref, } 1}(\mathbf{r}, t)$ and $H^{\text {ref, } 1}(\mathbf{r}, t)$. However, it could not be proved analytically that $\mathcal{G}^{\text {ref,2}}(\mathbf{r}, t)$, defined by Eq. (23), goes to zero as $t \rightarrow \infty$, although some performed numerical results do show that $\mathcal{G}^{\text {ref,2 }}(\mathbf{r}, t)$ goes to zero in the late time.
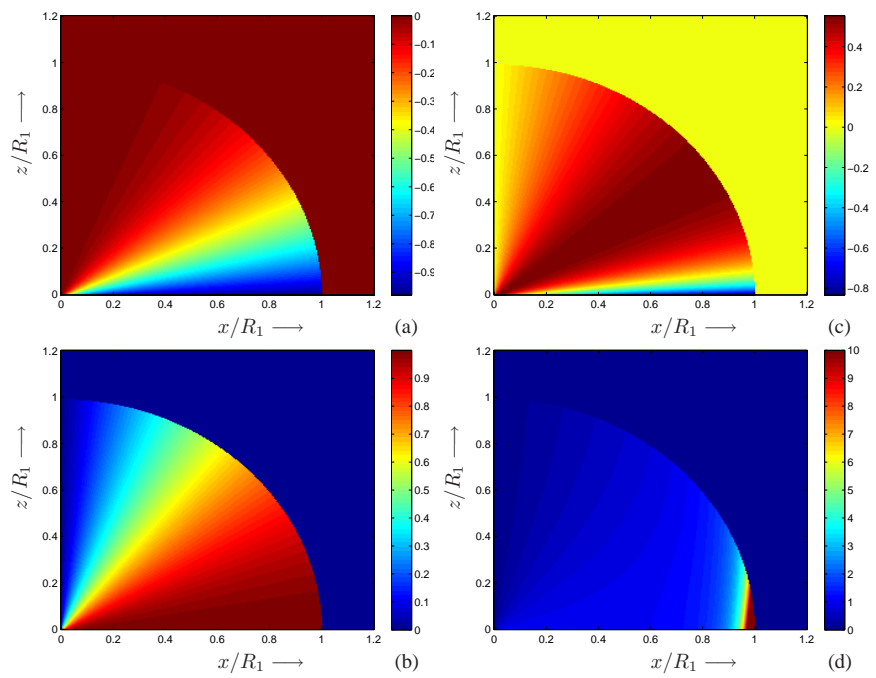

Fig. 4. Time snaps of the normalized RWGT components corresponding to $H_{y}^{\text {ref, } 1}(\mathbf{r}, t)$. (a) Far-zone RWGT component, $\left(4 \pi R_{1}^{2}\right) \times \mathcal{G}_{H_{y}}^{\text {ref }, 1 ; \mathrm{FF}}(\mathbf{r}, t)$, and (b) intermediate-zone RWGT component, $\left(4 \pi R_{1}^{2}\right) \times \mathcal{G}_{H_{y}}^{\mathrm{ref}, 1 ; \mathrm{IF}}(\mathbf{r}, t)$, for copper as the second medium. (c)-(d) Same as (a)-(b), but now for sea water as the second medium.

\section{CONCLUSIONS}

Space-time expressions for the impulsive wave fields generated by a vertical electric dipole located above a planar impedance boundary were derived. At the core of tackling the impedance boundary value problem corresponding to the 
elementary half-space configuration was the derivation of the space-time RWGF with the aid of the extended $\mathrm{CdH}$ method. The solutions for the reflected wave fields opened the door to analysis of their properties in the far-, intermediate-, and near-field regions, in the dependence of the second medium's electric conductivity and permittivity. Numerical results presented time snaps of the RWGT components to illustrate the spatial distribution of the reflected wave, and time traces of the measurable wave fields to illustrate the suitability of the presented analytical benchmark for general-purpose numerical methods.
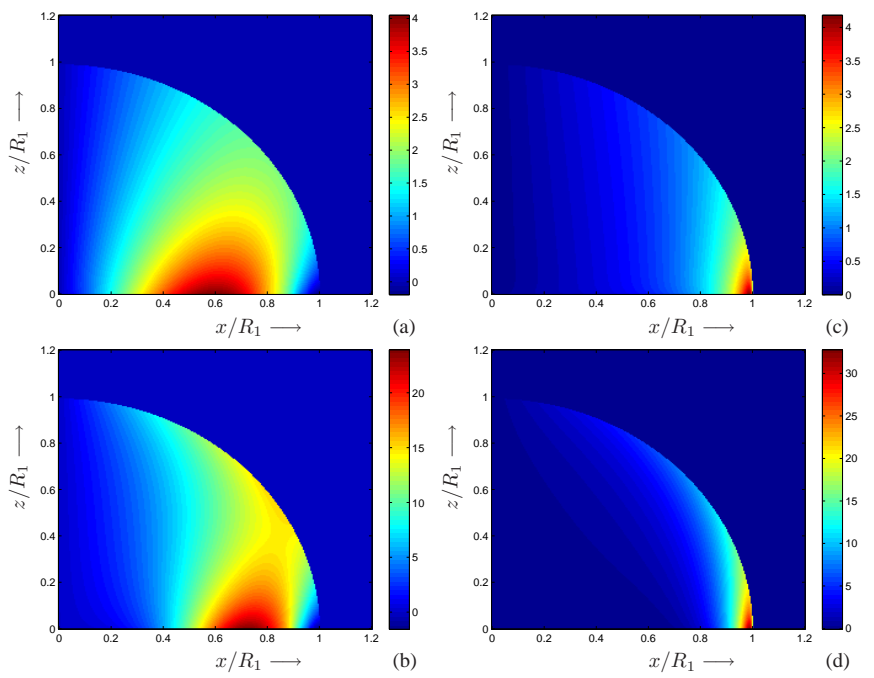

Fig. 5. Time snaps of the normalized RWGT components corresponding to $H_{y}^{\text {ref, }},(\mathbf{r}, t)$. Intermediate-zone RWGT component $\left(4 \pi R_{1}^{2}\right) \times \mathcal{G}_{H_{y}}^{\text {ref, }, 2 \mathrm{IF}}(\mathbf{r}, t)$ at (a) $t=\tau_{\text {rel }}$ and (b) $t=8 \tau_{\text {rel }}$ for copper as the second medium. (c)-(d) Same as (a)-(b), but now for sea water as the second medium.

\section{APPENDIX}

\section{A. The eXtended Cagniard-De Hoop Method}

The standard procedures in the $\mathrm{CdH}$ method, cf. [22], are carried out to furnish the transformation of the spaceLaplace RWGF in Eq. (16) back to the time domain. First, the transformations

$$
\begin{aligned}
\alpha & =-i p \cos (\theta)-q \sin (\theta), \\
\beta & =-i p \sin (\theta)+q \cos (\theta),
\end{aligned}
$$

are carried out. The vertical slowness becomes $\gamma(q, p)=$ $\left[\Omega(q)^{2}-p^{2}\right]^{1 / 2}$, with $\Omega(q)=\left(c_{1}^{-2}+q^{2}\right)^{1 / 2}$. Next, the integration path along the imaginary axis of the complex $p$ plane is replaced by the hyperbolic contour $p r+\gamma D_{z}=\tau$, with $D_{z}=z+h$ and $T_{1}(\mathbf{r}, q)<\tau<\infty$, where $T_{1}(\mathbf{r}, q)=$ $R_{1}(\mathbf{r}) \Omega(q)$. Note that $T^{\mathrm{ref}}=T^{\mathrm{ref}}(\mathbf{r})=T_{1}(\mathbf{r}, 0)$. Finally, the transformation

$$
q=\left(\tau^{2} / R_{1}^{2}-c_{1}^{-2}\right)^{1 / 2} \sin (\psi),
$$

is performed. These steps lead to the following equivalent of the space-Laplace RWGF in Eq. (16),

$$
\hat{\mathcal{G}}^{\mathrm{ref}}(\mathbf{r}, s)=\frac{1}{4 \pi R_{1}} \int_{\tau=T^{\mathrm{ref}}}^{\infty} \hat{L}^{\mathrm{ref}}(\mathbf{r}, \tau, s) \exp (-s \tau) \mathrm{d} \tau
$$

in which

$$
\begin{aligned}
\hat{L}^{\mathrm{ref}}(\mathbf{r}, \tau, s) & =\frac{2}{\pi} \int_{\psi=0}^{\pi / 2} \operatorname{Re}\{\tilde{R}(\bar{\gamma}, s)\} \mathrm{d} \psi \\
& =\frac{2}{\pi} \int_{\psi=0}^{\pi / 2} \operatorname{Re}\left\{1-\frac{2}{1+c_{1} \hat{\gamma}_{\mathrm{n}}(s)}\right\} \mathrm{d} \psi
\end{aligned}
$$

is the space-Laplace reflected-wave kernel function (RWKF), cf. [23], [24]. The definition for the reflection coefficient in Eq. (17) has been used. The vertical slowness is given by

$$
c_{1} \bar{\gamma}(\mathbf{r}, \tau, \psi)=\Gamma_{1}-\mathrm{i} \Gamma_{2} \cos (\psi),
$$

where $\Gamma_{1}(\mathbf{r}, \tau)$ and $\Gamma_{2}(\mathbf{r}, \tau)$ are given by Eqs. (21) and (22), respectively. If the time-domain counterpart of $\hat{L}^{\mathrm{ref}}(\mathbf{r}, \tau, s)$ is known, then the inverse Laplace transform of Eq. (51) is found as

$$
G^{\mathrm{ref}}(\mathbf{r}, t)=\frac{1}{4 \pi R_{1}} \int_{\tau=T^{\mathrm{ref}}}^{t} L^{\mathrm{ref}}(\mathbf{r}, \tau, t-\tau) \mathrm{d} \tau .
$$

The derivation of the space-time RWGF is hence reduced to finding the space-time RWKF $L^{\mathrm{ref}}(\mathbf{r}, \tau, t)$, which is discussed in Appendix B.
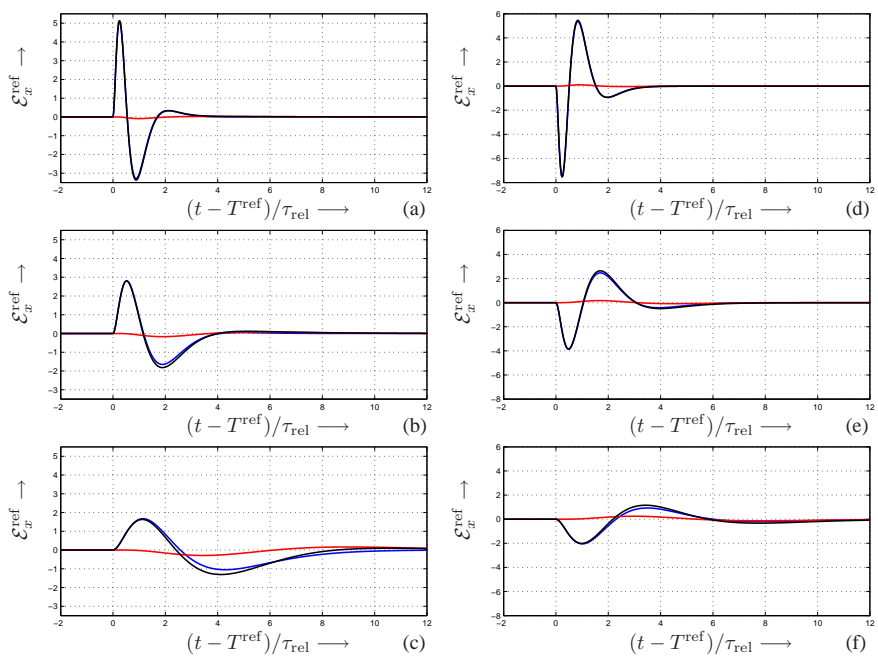

Fig. 6. Time traces of $\mathcal{E}_{x}^{\mathrm{ref}}(\mathbf{r}, t)$ at the position $(x, y, z)=(2 h, 0, h)$, with $h=2 c_{1} \tau_{\text {rel }}$, for copper as the second medium. The source signature's characterization time is (a) $\tau_{\mathrm{s}}=0.5 \tau_{\text {rel }}$, (b) $\tau_{\mathrm{s}}=\tau_{\text {rel }}$, and (c) $\tau_{\mathrm{s}}=2 \tau_{\text {rel }}$. The black and blue lines represent the modelled result for the total-admittance and constant-admittance reflected electric field strength, respectively. Their difference is the dispersive response of the material, which is represented by the red line. (d)-(f) Same as (a)-(c), but now for sea water as the second medium.

\section{B. SPACE-TIME REFLECTED-WAVE KERNEL FUNCTION}

The time-domain counterpart of the space-Laplace RWKF $\hat{L}^{\mathrm{ref}}(\mathbf{r}, \tau, s)$ in Eq. (52) is here derived with the aid of the Schouten-Van der Pol theorem [25], [26], [27], [28]. First, Eq. (52) is written as

$$
\hat{L}^{\mathrm{ref}}(\mathbf{r}, \tau, s)=1-\frac{2}{\pi} \int_{\psi=0}^{\pi / 2} \operatorname{Re}\left\{\frac{2}{A-\mathrm{i} B \cos (\psi)}\right\} \mathrm{d} \psi,
$$

in which $A(\mathbf{r}, \tau, s)=1+\Gamma_{1} \hat{Y}_{\mathrm{n}}(s)$ and $B(\mathbf{r}, \tau, s)=\Gamma_{2} \hat{Y}_{\mathrm{n}}(s)$. Evaluation of the $\psi$-integral with the aid of the identity

$$
\frac{2}{\pi} \int_{\psi=0}^{\pi / 2} \frac{A}{A^{2}+B^{2} \cos ^{2}(\psi)} \mathrm{d} \psi=\frac{1}{\left(A^{2}+B^{2}\right)^{1 / 2}},
$$


yields

$$
\hat{L}^{\mathrm{ref}}(\mathbf{r}, \tau, s)=1-\frac{2 \Lambda_{3}}{\left\{\left[\hat{Y}_{\mathrm{n}}(s)+\Lambda_{1}\right]^{2}+\Lambda_{2}^{2}\right\}^{1 / 2}},
$$

in which $\Lambda_{1}(\mathbf{r}, \tau), \Lambda_{2}(\mathbf{r}, \tau)$ and $\Lambda_{3}(\mathbf{r}, \tau)$ are given by Eqs.(26)-(28). To recast the term on the r.h.s. of Eq. (57) to an appropriate form, the Schouten-Van der Pol theorem (cf. Formula 29.3.55 in [32]) is invoked at this stage, in conjunction with elementary rules of the Laplace transform. The result is

$$
\frac{2 \Lambda_{3}}{\left\{\left[\hat{Y}_{\mathrm{n}}(s)+\Lambda_{1}\right]^{2}+\Lambda_{2}^{2}\right\}^{1 / 2}}=2 \int_{v=0}^{\infty} K_{\mathrm{C}}(\mathbf{r}, \tau, v) \hat{K}_{\mathrm{Y}}(v, s) \mathrm{d} v,
$$

in which $K_{\mathrm{C}}(\mathbf{r}, \tau, v)$ is given by Eq. (25) and

$$
\hat{K}_{\mathrm{Y}}(v, s)=\exp \left[-v \hat{Y}_{\mathrm{n}}(s)\right],
$$

is a kernel function that contains only the second medium's EM parameters via the specific wave admittance. With the help of Eq. (58), the space-Laplace RWKF in Eq. (57) is reshaped to the form

$$
\hat{L}^{\mathrm{ref}}(\mathbf{r}, \tau, s)=1-2 \int_{v=0}^{\infty} K_{\mathrm{C}}(\mathbf{r}, \tau, v) \hat{K}_{\mathrm{Y}}(v, s) \mathrm{d} v .
$$

If the time-domain counterpart of $\hat{K}_{\mathrm{Y}}(v, s)$ is known, then the inverse Laplace transform of Eq. (60) is found as

$$
\hat{L}^{\mathrm{ref}}(\mathbf{r}, \tau, t)=\delta(t)-2 \int_{v=0}^{\infty} K_{\mathrm{C}}(\mathbf{r}, \tau, v) K_{\mathrm{Y}}(v, t) \mathrm{d} v .
$$

The derivation of the space-time RWKF is hence reduced to finding $K_{\mathrm{Y}}(v, t)$, which is the time-domain counterpart of Eq. (59). The procedures are discussed in Appendix C.
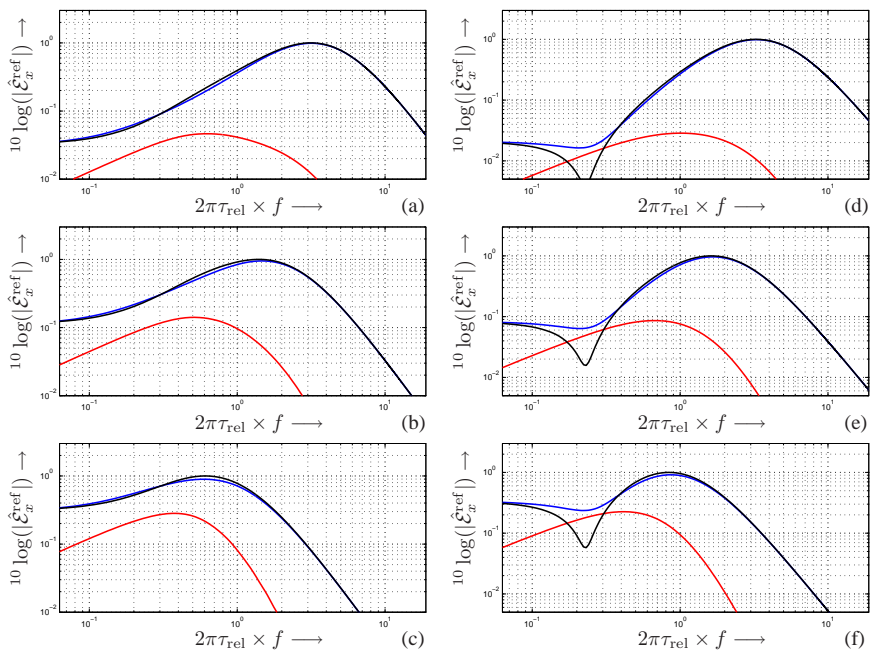

Fig. 7. Normalized magnitude spectrum of $\mathcal{E}_{x}^{\text {ref }}(\mathbf{r}, t)$ at the position $(x, y, z)=(2 h, 0, h)$, with $h=2 c_{1} \tau_{\text {rel }}$, for copper as the second medium. The source signature's characterization time is (a) $\tau_{\mathrm{s}}=0.5 \tau_{\text {rel }}$, (b) $\tau_{\mathrm{s}}=\tau_{\text {rel }}$, and (c) $\tau_{\mathrm{s}}=2 \tau_{\text {rel }}$. The black and blue lines correspond to the total-admittance and constant-admittance reflected electric field strength, respectively. Their difference is the dispersive response of the material, which is represented by the red line. (d)-(f) Same as (a)-(c), but now for sea water as the second medium.

\section{TRANSFORMATION BACK TO THE TIME DOMAIN}

The analytical transformation of the kernel function $\hat{K}_{\mathrm{Y}}(v, s)$ back to the time domain is here discussed. With the help of Eq. (18), Eq. (59) is first written as

$$
\hat{K}_{\mathrm{Y}}(v, s)=\exp \left[-v \hat{X}_{\mathrm{Y}}^{1 / 2}(s)\right] .
$$

Next, the Schouten-Van der Pol theorem (c.f. Formula 29.3.82 in [32]) is invoked to express the r.h.s. of Eq. (62) as an integral representation. This yields

$$
\exp \left[-v \hat{X}_{\mathrm{Y}}^{1 / 2}(s)\right]=\int_{w=0}^{\infty} \Psi(v, w) \exp \left[-w \hat{X}_{\mathrm{Y}}(s)\right] \mathrm{d} w
$$

in which $\Psi(v, w)$ is given by Eq. (24). The r.h.s. of Eq. (63) is in an appropriate form for finding $K_{\mathrm{Y}}(v, t)$ analytically. The time-domain counterpart of $\hat{K}_{\mathrm{Y}}(v, s)$ is first expressed as

$$
\begin{aligned}
& K_{\mathrm{Y}}(v, t)=\mathcal{L}^{-1}\left\{\exp \left[-v \hat{X}_{\mathrm{Y}}^{1 / 2}(s)\right]\right\}= \\
& \int_{w=0}^{\infty} \Psi(v, w) \exp \left(-F^{2} w\right) \mathcal{L}^{-1}\left\{\exp \left(-w F^{2} \tau_{\text {rel }}^{-1} s^{-1}\right)\right\} \mathrm{d} w
\end{aligned}
$$

in which $\mathcal{L}^{-1}$ stands for the inverse Laplace transformation. With the aid of the identity (cf. Formula 29.3.75 in [32]),

$$
\begin{aligned}
& s^{-1} \exp \left(-k s^{-1}\right)= \\
& \int_{t=0}^{\infty} \exp (-s t)\left\{J_{0}\left[2(k t)^{1 / 2}\right] H(t)\right\} \mathrm{d} t, \quad k \geq 0,
\end{aligned}
$$

$K_{\mathrm{Y}}(v, t)$ is explicitly found as

$$
\begin{aligned}
& K_{\mathrm{Y}}(v, t)=\int_{w=0}^{\infty} \Psi(v, w)\left\{\exp \left(-F^{2} w\right) \delta(t)-\right. \\
& \left.F \exp \left(-F^{2} w\right)\left(\frac{w \tau_{\text {rel }}^{-1}}{t}\right)^{1 / 2} J_{1}\left[2 F\left(w t \tau_{\text {rel }}^{-1}\right)^{1 / 2}\right] H(t)\right\} \mathrm{d} w .
\end{aligned}
$$

Equations (54), (61) and (66) complete the solution for the space-time reflected-wave Green's function.
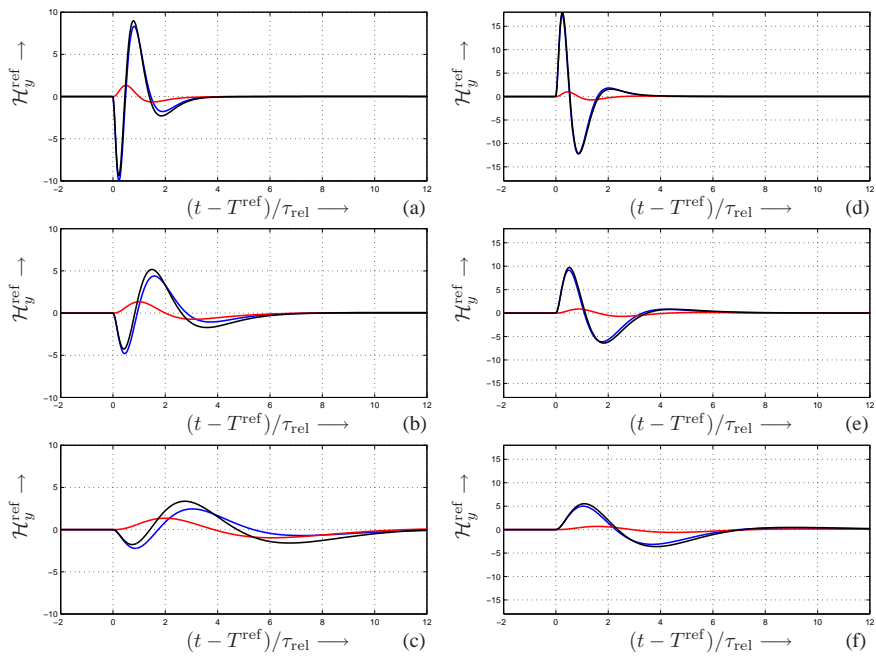

Fig. 8. Time traces of $\mathcal{H}_{y}^{\mathrm{ref}}(\mathbf{r}, t)$ at the position $(x, y, z)=(2 h, 0, h)$, with $h=2 \tau_{\text {rel }} c_{1}$, for copper as the second medium. The source signature's characterization time is (a) $\tau_{\mathrm{s}}=0.5 \tau_{\text {rel }}$, (b) $\tau_{\mathrm{s}}=\tau_{\text {rel }}$, and (c) $\tau_{\mathrm{s}}=2 \tau_{\text {rel }}$. (d)-(f) Same as (a)-(c), but now for sea water as the second medium. 


\section{WAVE FIELDS IN HOMOGENEOUS MEDIA}

Let $R_{0}(\mathbf{r})=\left(x^{2}+y^{2}+|z-h|^{2}\right)^{1 / 2}>0$ denote the distance between source and receiver, and $T^{\text {inc }}(\mathbf{r})=R_{0}(\mathbf{r}) / c_{1}$ the arrival time of the incident wave. The incident-wave Green's function or just the Green's function in homogeneous media is given by $\mathcal{G}^{\text {inc }}(\mathbf{r}, t)=G^{\text {inc }}(\mathbf{r}) H\left[t-T^{\text {inc }}(\mathbf{r})\right]$, in which $G^{\text {inc }}(\mathbf{r})=1 / 4 \pi R_{0}$. The wave field expressions corresponding to an electric dipole with orientation in the $z$-direction are given by (e.g. [30])

$$
\begin{aligned}
E_{x}^{\mathrm{inc}}(\mathbf{r}, t)= & \varepsilon_{1}^{-1} J(t) \stackrel{(t)}{*} \partial_{x} \partial_{z} \mathcal{G}^{\mathrm{inc}}(\mathbf{r}, t), \\
E_{y}^{\mathrm{inc}}(\mathbf{r}, t)= & \varepsilon_{1}^{-1} J(t) \stackrel{(t)}{*} \partial_{y} \partial_{z} \mathcal{G}^{\mathrm{inc}}(\mathbf{r}, t), \\
E_{z}^{\mathrm{inc}}(\mathbf{r}, t)= & \varepsilon_{1}^{-1} J(t) \stackrel{(t)}{*} \partial_{z}^{2} \mathcal{G}^{\mathrm{inc}}(\mathbf{r}, t) \\
& +\mu_{1} \partial_{t}^{2} J(t) \stackrel{(t)}{*} \mathcal{G}^{\mathrm{inc}}(\mathbf{r}, t), \\
H_{x}^{\mathrm{inc}}(\mathbf{r}, t)= & +\partial_{t} J(t) \stackrel{(t)}{*} \partial_{y} \mathcal{G}^{\mathrm{inc}}(\mathbf{r}, t), \\
H_{y}^{\mathrm{inc}}(\mathbf{r}, t)= & -\partial_{t} J(t) \stackrel{(t)}{*} \partial_{x} \mathcal{G}^{\mathrm{inc}}(\mathbf{r}, t), \\
H_{z}^{\text {inc }}(\mathbf{r}, t)= & 0 .
\end{aligned}
$$

These expressions are equivalent to the expressions in Eqs. (10)-(15) (apart from a minus sign difference between the incident and reflected electric field strength). As a result, the expressions for the reflected wave field and RWGTs in Sect. IV can be checked by deriving directly the well-known solutions for the EM wave fields in homogeneous media from Eqs. (29)-(41). Only $E_{x}^{\text {inc }}(\mathbf{r}, t)$ and $H_{y}^{\text {inc }}(\mathbf{r}, t)$ are investigated, the other components can be derived similarly. First, $\partial_{x} G^{\text {inc }}(\mathbf{r})=-x / 4 \pi R_{0}^{3}, \partial_{z} G^{\text {inc }}(\mathbf{r})=-(z-h) / 4 \pi R_{0}^{3}$ and $\partial_{x} \partial_{z} G^{\text {inc }}(\mathbf{r})=x(z-h) / 4 \pi R_{0}^{5}$ are substituted in Eqs. (32)(34) and Eqs. (39)-(40), where $D_{z}$ is replaced by $(z-h)$. The results are then substituted in Eqs. (30) and (37), respectively. The result for $E_{x}^{\text {inc }}(\mathbf{r}, t)$ has to be corrected for a minus sign due to the minus sign difference between Eqs. (67) and (10). The solutions for $E_{x}^{\text {inc }}(\mathbf{r}, t)$ and $H_{y}^{\text {inc }}(\mathbf{r}, t)$ are easily derived, and arranged as

$$
\begin{gathered}
E_{x}^{\mathrm{inc}}(\mathbf{r}, t)=\frac{x(z-h)}{4 \pi R_{0}^{5} \varepsilon_{1}}\left(T^{\mathrm{inc}}\right)^{2} \partial_{t} J\left[t-T^{\mathrm{inc}}(\mathbf{r})\right] \\
+\frac{3 x(z-h)}{4 \pi R_{0}^{5} \varepsilon_{1}} T^{\mathrm{inc}} J\left[t-T^{\mathrm{inc}}(\mathbf{r})\right] \\
+\frac{3 x(z-h)}{4 \pi R_{0}^{5} \varepsilon_{1}} \int_{u=0}^{t-T^{\mathrm{inc}}} J(u) \mathrm{d} u,
\end{gathered}
$$

for $t \geq T^{\text {inc }}(\mathbf{r})$, and $E_{x}^{\text {inc }}(\mathbf{r}, t)=0$ for $t<T^{\text {inc }}(\mathbf{r})$, and

$$
\begin{array}{r}
H_{y}^{\mathrm{inc}}(\mathbf{r}, t)=\frac{x}{4 \pi R_{0}^{3}} T^{\mathrm{inc}} \partial_{t} J\left[t-T^{\mathrm{inc}}(\mathbf{r})\right] \\
+\frac{x}{4 \pi R_{0}^{3}} J\left[t-T^{\mathrm{inc}}(\mathbf{r})\right]
\end{array}
$$

for $t \geq T^{\text {inc }}(\mathbf{r})$, and $H_{y}^{\text {inc }}(\mathbf{r}, t)=0$ for $t<T^{\text {inc }}(\mathbf{r})$. The expressions given by Eqs. (73) and (74) are the solutions for the EM wave fields in homogeneous media and, consequently, show that Eqs. (29)-(41) are consistent with the solutions for this special case.
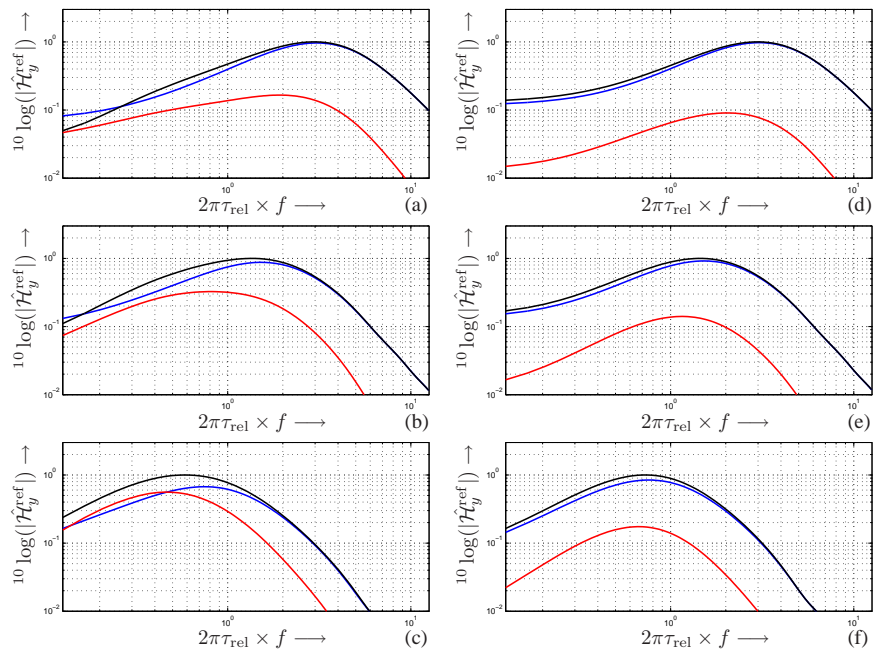

Fig. 9. Normalized magnitude spectrum of $\mathcal{H}_{y}^{\mathrm{ref}}(\mathbf{r}, t)$ at the position $(x, y, z)=(2 h, 0, h)$, with $h=2 \tau_{\text {rel }} c_{1}$, for copper as the second medium. The source signature's characterization time is (a) $\tau_{\mathrm{s}}=0.5 \tau_{\mathrm{rel}}$, (b) $\tau_{\mathrm{s}}=\tau_{\text {rel }}$, and (c) $\tau_{\mathrm{s}}=2 \tau_{\text {rel }}$. (d)-(f) Same as (a)-(c), but now for sea water as the second medium.

\section{REFERENCES}

[1] M. A. Leontovich, "Methods of solution for problems of electromagnetic waves propagation along the Earth surface," Bull. Acad. Sci. USSR, Phys. Ser. vol. 8, no. 1, p 1622, 1944, (in Russian).

[2] A. N. Shchukin, Propagation of Radio Waves. Moscow, Russia: Svyazizdat, 1940.

[3] S. W. Lee and W. Gee, "How good is the impedance boundary condition?," IEEE Trans. Antennas Propag., vol. 35, no. 11, pp. 1313$1315,1987$.

[4] D. J. Hoppe and Y. Rahmat-Samii, Impedance Boundary Conditions in Electromagnetics. Washington, DC: Taylor \& Francis, 1995.

[5] I. V. Lindell and A. H. Sihvola, "Realization of impedance boundary," IEEE Trans. Antennas Propag., vol. 54, no. 12, pp. 3669-3676, 2006.

[6] A. Karlsson, "Approximate boundary conditions for thin structures," IEEE Trans. Antennas Propag., vol. 57, no. 1, pp. 144-148, 2009.

[7] J. Jin, The Finite Element Method in Electromagnetics. Wiley-IEEE Press, 2nd ed., 2002.

[8] A. Taflove and S. C. Hagness, Computational Electrodynamics: The Finite-Difference Time-Domain Method. Artech House Publishers, 3rd ed., 2005.

[9] K. S. Oh and J. E. Schutt-Aine, "An efficient implementation of surface impedance boundary conditions for the finite-difference time-domain method," IEEE Trans. Antennas Propag., vol. 43, no. 7, pp. 660666, 1995.

[10] D. V. Thiel and R. Mittra, "Surface impedance modelling using the finite-difference time-domain method," IEEE Trans. Geosci. Remote Sens., vol. 35, no. 5, pp. 1350-1356, 1997.

[11] R. F. Harrington and J. R. Mautz, "An impedance sheet approximation for thin dielectric shells," IEEE Trans. Antennas Propag., vol. 23, no. 7, pp. 531-534, 1975.

[12] R. J. Luebbers and K. Kunz, "FDTD modelling of thin impedance sheets," IEEE Trans. Antennas Propag., vol. 40, pp. 349-351, 1992.

[13] T. B. Senior, "Approximate boundary conditions," IEEE Trans. Antennas and Propag., vol. 29, pp. 826-829, 1981.

[14] S. van den Berghe, F. Olyslager, and D. de Zutter, "Accurate modelling of thin conducting layers in FDTD," IEEE Microw. Guided Wave Lett., vol. 8, no. 2, pp. 75-77, 1998.

[15] J. C. Rautio and V. Demir, "Microstrip conductor loss models for electromagnetic analysis," IEEE Trans. Microwave Theory Tech., vol. 28, no. 3, pp. 434-444, 2005.

[16] M.-Y. Xia, C. H. Chan, and W. C. Chew, "Time-domain Green's functions for the microstrip structures using Cagniard-de Hoop method," IEEE Trans. Antennas and Propag., vol. 52, no. 6, pp. 1578-1585, 2004.

[17] K. M. Coperich, A. E. Ruehli and A. C. Cangellaris, "Enhanced skin effect for partial-element equivalent-circuit (PEEC) models," IEEE Trans. Microwave Theory Tech., vol. 48, no. 9, pp. 1435-1442, 2000.

[18] D. de Zutter, H. Rogier, L. Knockaert, and J. Sercu, "Surface current modelling of the skin effect for on-chip interconnects," IEEE Trans. Adv. Pack., vol. 30, no. 2, pp. 342-349, 2007. 
[19] A. Rong, A. C. Cangellaris, and L. Dong, "Comprehensive broadband electromagnetic modelling of on-chip interconnect with a surface discretization-based generalized PEEC model," IEEE Trans. Adv. Pack., vol. 28, no. 3, pp. 434-444, 2005.

[20] I. V. Lindell and E. Alanen, "Exact image theory for the Sommerfel half-space problem, II, Vertical electric dipole," IEEE Trans. Antennas Propag., vol. 32, no. x, pp. 841-847, 1984.

[21] B. J. Kooij, "The electromagnetic field emitted by a pulsed current point source above the interface of a nonperfectly conducting Earth," Radio Science, vol. 31, no. 6, pp. 1345-1360, 1996.

[22] A. T. de Hoop and H. J. Frankena, "Radiation of pulses generated by a vertical electric dipole above a plane, nonconducting, Earth," Appl. Sci. Res., Sect. B, vol. 8, pp. 369-377, 1960.

[23] C. H. Lam, B. J. Kooij, and A. T. de Hoop, "Impulsive sound reflection from an absorptive and dispersive planar boundary," J. Acoust. Soc. Am., vol. 116, no. 2, pp. 677-685, 2004.

[24] A. T. de Hoop, C. H. Lam and B. J. Kooij, "Parametrization of acoustic boundary absorption and dispersion properties in time-domain source/receiver reflection measurement," J. Acoust. Soc. Am., vol. 118, no. 2, pp. 654-660, 2005.

[25] J. P. Schouten "A new theorem in operational calculus together with an application of it," Physica (Amsterdam), 1, pp. 75-80, 1934.

[26] B. van der Pol, "A theorem on electrical networks with applications to filters," Physica (Amsterdam), 1, pp. 521-530, 1934.

[27] B. van der Pol and H. Bremmer Operational Calculus Based on the Two-sided Laplace Transform. Cambridge University Press, Cambridge, UK, pp. 232-236, 1950.

[28] J. P. Schouten, Operatorenrechnung. Springer, Berlin, pp. 124126, 1961.

[29] D. V. Widder, The Laplace Transform. New Jersey: Princeton University Press, pp. 61-63, 1946.

[30] W. C. Chew, Waves and Fields in Inhomogeneous Media (IEEE Press Series on Electromagnetic Waves), 1995.

[31] W. A. Strauss, Partial Differential Equations: An Introduction. John Wiley \& Sons, Inc., New York, USA, Sect. 2.3, 1992.

[32] M. Abramowitz and I. A. Stegun, Handbook of Mathematical Functions. Dover Publications, Inc., New York, USA, p 1020, 1972. 